\title{
Theoretical Analysis on the Spectral Density of Random Slivers
}

\author{
By Kiyohisa Fujino and Sueo Kawabata, MEMBERs, TMSJ \\ Faculty of Engineering, Kyoto University, Kyoto
}

\begin{abstract}
This article concerns d theoretical analysis of random slivers. The assumptions made in it are that all fibers are straight and parallel to the sliver axis; that each single fiber is of a constant thickness; and that fibers are arranged at random according to some statistic process or other.

The authors have formulated a function which expresses the thickness of slivers, to begin with, and have calculated its spectral density by Fourier transformation. Then, as a special case in the general formula, the so-called "random slivers" are denoted and the characteristics of their irregularity explained.

The results of this analysis make it possible to explain an empirical fact, such as that the most conspicuous wave length component contained in the irregularity of a normal yarn is $2 L$ when the constituent fibers are of uniform Iength $L$.

This theory can be used to solve problems attending automatic control of sliver levelness, the technique of the measurement of sliver or yarn irregularity, the drafting and the theory of blending.
\end{abstract}

\section{Introduction}

This paper describes a theoretical analysis of the fundamental characteristics of sliver irregularity. Fibers in an "actual" sliver are not arranged systematically as in an "ideal sliver" in which the leading ends of the fibers are distributed uniformly as shown in Figure 1. This irregular arrangement of fibers produces thickness variations of slivers.

No matter how often doubling and ideal drafting are repeated, a certain thickness irregularity of slivers remains because of the statistic properties of the random arrangement of parallel fibers. [1]

Sliver thickness has come up for discussion as an object of automatic control, and a strong need has arisen for comprehensive data about the properties of sliver irregularity in the designing of an automatic control system.

Appart from this practical reqirement, a clarification of the fundamental characteristics of sliver irregularity is important because it is a vital part of the spinning theory.

The present article begins with consideration of the statistic characteristics of sliver irregularity where fibers are arranged according to some statistic process in a sliver. Then it seeks to derive a general formula to describe the characteristics of this irregularity.

The article goes on to consider the construction of random slivers and deduce the characteristics of sliver thickness irregularity by following the general

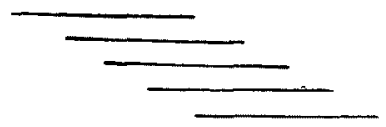

Fig. 1 Fiber arrangement in "Ideal sliver." formula.

The spectrum of variance (the square of the mean deviation) corresponding to wave length is used here to describe the characteristics of irregularity, this spectrum being deduced directly from the spectral density of thickness irregularity.

Many studies have been made about the drafting process with the ideal sliver (shown in Figure 1) used frequently as an ideal model of what actual slivers should be. However, the ideal state of an actual sliver is the state not of an "ideal sliver" but of a "random sliver" in which the fibers are distributed at random. The theory deduced in this article may be useful in constructing a drafting theory based on random slivers.

Again, the results of the present study can be applied to the solution of the problems concering the technique of the measurement of yarn or sliver irregularity, automatic control of sliver thickness and the theory of blending.

\section{Fundamental Theory}

1-1. "Sliver Function" as the Representation of Sliver Thickness for Mathematical Analysis

To consider a sliver theoretically, we have to elicit a mathematically equivalent representation which describes thickness in the cross section of a sliver.

Assume that all fibers are straight and parallel to the sliver axis and the thickness of a single fiber is constant. Then, consider a fiber of length $l_{i}$, with its leading end at place $x_{i}$ on the sliver axis as shown in Figure 2.

Put a rectangular impulse as the equivalent function describing the single fiber, because this im- 
pulse function can represent the thickness of the fiber and its position in the sliver. Let us term this equivalent function a "single fiber function."

Since a sliver is constructed of many fibers, the thickness in the cross section can be expressed by the assembly of the "single fiber functions," i.e., the assembly of the impulse functions shown in Figure 3.

Let the function $a U_{i}(x)$ be the rectangular impulse function termed the "single fiber function," where $a$ is the thickness of the single fiber and assumed to be constant, and $U_{i}(x)$ is the rectangular impulse of a unit height which begins at $x_{i}$. That is:

$$
a U_{i}(x)=\left\{\begin{array}{l}
0 \text { for } x_{i}>x, \quad x_{i}+l_{i}<x \\
a \text { for } x_{i}<x<x_{i}+l_{i}
\end{array}\right.
$$

where $x_{i}$ : position of the leading end of the fiber

$l_{i}$ : length of the fiber

a: thickness of a single fiber

The thickness of the sliver is described as an assembly of these impulses. Let $S(x)$ be the thickness of the sliver at place $x$ on the sliver axis, then we get

$$
S(x)=\sum_{i=-N}^{N} a U_{i}(x)
$$

where $\mathcal{N}$ : positive integer

Let the function $S(x)$ be a "sliver function," $S(x)$ is the mathematically equivalent function to sliver irregularity.

1-2. Definitions of the Spectral Density Function and the Spectrum of the Variance $\sigma^{2}$ CorresPonding to Wave Length

Generally, let $f(x)$ be a stationary random function. Then the spectral density function $Y(\omega)$ is defined as follows:

Put

$$
A(\omega)=\int_{-\infty}^{\infty} f(x) e^{-j \omega x} d x
$$

then,

$$
Y(\omega)=\lim _{X \rightarrow \infty} \frac{1}{X}|A(\omega)|^{2}
$$

Equivalent function

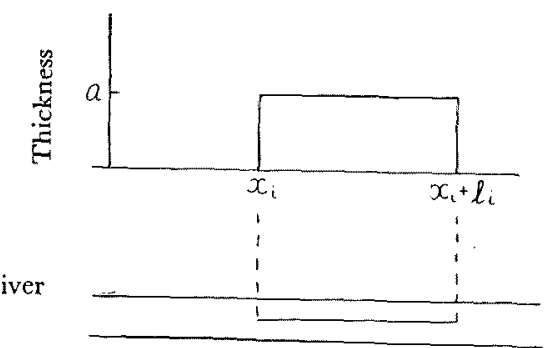

Fig. 2 A single fiber in a sliver and its equivalent represen-
tation. where $\omega=2 \pi / p$, and $p:$ wave length

$A(\omega)$ : Fourier transform of $f(x)$ and proportional to the Fourier spectrum.

Let the variance (square of the standard deviation) of $f(x)$ be $\sigma^{2}$. Then $\sigma^{2}$ is deduced from the spectral density function $r(\omega)$, as follows:

$$
\sigma^{2}=\frac{1}{2 \pi} \lim _{\omega \rightarrow 0} \int_{\omega}^{\infty} Y(\omega) d \omega
$$

Thus, variance $\sigma^{2}$ can be deduced easily from the spectral density function.

Let $X(p)$ be the distribution function of variance $\sigma^{2}$ corresponding to wave length $p$. Then $X(p)$ is deduced from formula (5), if the spectral density function $r(\omega)$ is known. That is:

$$
X(p)=\frac{1}{p^{2}} Y\left(\frac{2 \pi}{p}\right)
$$

In other words, the function $X(p)$ is the spectrum of variance $\sigma^{2}$ corresponding to wave length $p$.

For instance, let $\sigma^{2}\left(p_{1}, p_{2}\right)$ be the variance of a variation which contains the wave length component from $p_{1}$ to $p_{2}$. Then, $\sigma^{2}\left(p_{1} . p_{2}\right)$ is indicated as follows:

$$
\sigma^{2}\left(p_{1}, p_{2}\right)=\int_{p_{x}}^{p_{x}} X(p) d p
$$

This integration is shown by the shaded area in Figure 4. Hence, the total area under $X(p)$ curve is equal to the variance $\sigma^{2}$ of stationary random function $f(x)$.

In this article, function $X(p)$ is deduced from the spectral density function and used to characterize the irregularity of slivers.

Equivalent function

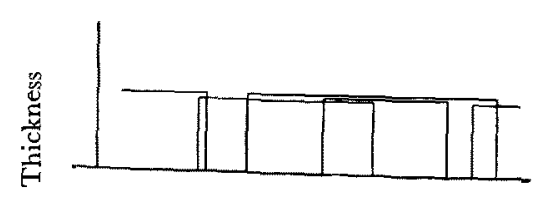

Sliver

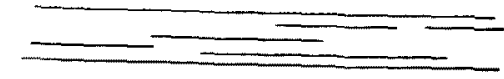

Fig. 3 "Sliver" function

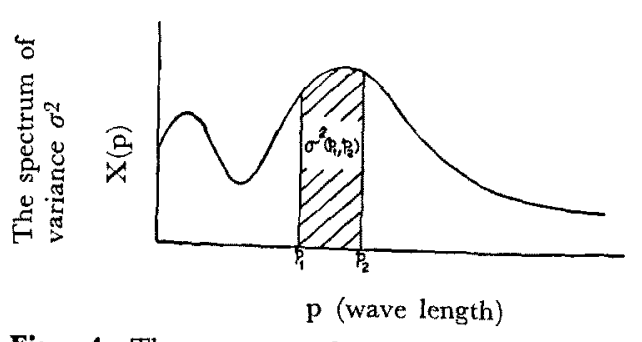

Fig. 4 The spectrum of variance $\sigma^{2}$ corresponding to wave length. Total area under the $\mathrm{X}(\mathrm{p})$ curve is equal to variance $\sigma^{2}$. 
1-3. Calculation of the Spectral Density of "Sliver Function"

Assume the sliver function $S(x)$ to be a stationary random function. And substituting formula (2) into (3), we get:

$$
A(\omega)=\int_{-\infty}^{\infty} a \sum_{i=-N}^{N} U_{i}(x) e^{-j \omega x} d x
$$

For the calculation of the integral in formula (8) the technique of Laplace transformation comes in handy. Let $H(x)$ be the heaviside unit function, and then $U_{i}(x)$ is represented as follows:

$$
U_{i}(x)=H\left(x-x_{i}\right)-H\left(x-x_{i}+l_{i}\right)
$$

Substituting formula (9) into formula (8), we get:

$$
\begin{aligned}
A(\omega)= & a \sum_{i=-N}^{N} \int_{x_{l}}^{x_{i}+l_{l}}\left\{H\left(x-x_{i}\right)\right. \\
& \left.-H\left(x-x_{i}+l_{i}\right)\right\} e^{-j \omega x} d x
\end{aligned}
$$

The above integration results in:

$$
A(\omega)=a \sum_{i=-N}^{N} \frac{1}{j \omega} e^{-j \omega x_{t}}\left(1-e^{-j \omega t}\right)
$$

From formulas (4) and (11), the spectral density function $Y(\omega)$ is formed thus:

$$
\begin{aligned}
Y(\omega)= & \frac{a^{2}}{\pi \bar{\varepsilon} \omega^{2}} \lim _{N \rightarrow \infty} \frac{1}{2 N} \sum_{i=-N+1, k=-N+1}^{N} \sum^{N} e^{-j \omega x_{t}} \\
& \times e^{-j \omega x_{k}}\left(1-e^{-j \omega t k}\right)\left(1-e^{j \omega / k}\right) \cdots \cdots
\end{aligned}
$$

Assuming that $x_{i}$ and $l_{i}$ are uncorrelated, we get the following final result with $\mathcal{N} \rightarrow \infty$ :

$$
\begin{aligned}
Y(\omega) & =\frac{2 a^{2}}{\varepsilon \omega^{2}}\left\{2-Z(\omega)-Z^{*}(\omega)\right. \\
& +2(1-Z(\omega))\left(1-Z^{*}(\omega)\right) \cdot \operatorname{Re} \sum_{i=1}^{\infty}[K(\omega)\}^{t}
\end{aligned}
$$

where $\varepsilon=x_{i}+{ }_{1}-x_{i}$, i.e., the distance between the leading ends of fibers (see Figure 5), and $\bar{\varepsilon}$ is the mean values of $\varepsilon$.

$Z^{*}(\omega)$ : the complex conjugate of $Z(\omega)$ and $Z(\omega)$ is defined by formula (15).

$R e \quad:$ this symbol means "the real part of." $K(\omega)$ and $Z(\omega)$ are the characteristic functions [2]

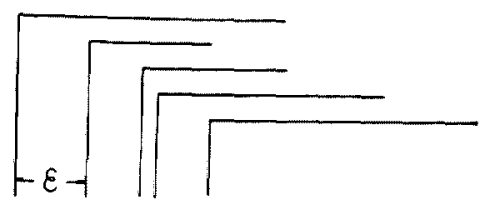

Fig. 5 Distance between the leading ends of fibers. of $\varepsilon$ and fiber length $l$, respectively, and are defined as follows:

$$
K(\omega)=\int_{0}^{\infty} P(\varepsilon) e^{-j \omega \varepsilon} d \varepsilon
$$

where $P(\varepsilon)$ : the probability distribution function of $\varepsilon$.

$$
Z(\omega)=\int_{0}^{\infty} P(l) e^{-j \omega t} d l
$$

where $P(l)$ : the probability distribution function of fiber length $l$.

Formula (13) can be rewritten as follows when $|K(\omega)|<1$ :

$$
\begin{aligned}
Y(\omega)= & \frac{4 a^{2}}{\bar{\varepsilon} \omega^{2}}\{1-\operatorname{Re} Z(\omega)+(1-Z(\omega)) \\
& \left.\times\left(1-Z^{*}(\omega)\right) \operatorname{Re} \frac{K(\omega)}{1-K(\omega)}\right\} \cdots
\end{aligned}
$$

Put the real part and the imaginary part of $K(\omega)$ to be $\varphi(\omega)$ and $\psi(\omega)$ respectively. Then:

$$
K(\omega)=\phi(\omega)+j \psi(\omega)
$$

Formula (16) can now be rewritten as follows:

$$
\begin{aligned}
Y(\omega) & =\frac{4 a^{2}}{\bar{\varepsilon} \omega^{2}}\{1-\operatorname{Re} Z(\omega)+(1-Z(\omega)) \\
& \left.\times\left(1-Z^{*}(\omega)\right) \frac{\phi(\omega)-\phi^{2}(\omega)-\psi^{2}(\omega)}{(1-\phi(\omega))^{2}+\psi^{2}(\omega)}\right\}
\end{aligned}
$$

Formula (13) (or (16), (17) ) is the general formula which determines the characteristics of thickness irregularity when fibers are arranged according to a specified statistic distribution in slivers. Given the fiber length distribution, the mean thickness of slivers, the thickness of single fibers and specified statistic properties of the fiber arrangement in a sliver, we can directly calculate the spectral density function $\Upsilon(\omega)$ by using the general formula.

\section{1-4. Spectral Density Function of "Random Slivers"}

To deduce the spectral density function of "random slivers" by the general formula (13) (or (16), (17) ), we have to know the statistic characteristics of the fiber arrangement in "random slivers" (that is, the characteristic function $K(\omega)$ has to be determined, where function $K(\omega)$ is deduced from formula (14) if $P(\varepsilon)$ is known).

Let $n$ be the number of the leading ends of fibers per unit sliver length, and $P(n)$ be the probability distribution function of $n$.

To find out the distribution function $P(\varepsilon)$, assume the function $P(n)$ to be Poisson's distribution function. That is: 


$$
P(n)=\frac{e^{-m} m^{n}}{n !}
$$

where $m$ : the mean value of $n$.

Then, $P(\varepsilon)$ is deduced from $P(n)$ by solving the following integral equation:

$$
C e^{-X}=\int_{X}^{\infty} P(\varepsilon) d \varepsilon
$$

where $C$ : constant value.

The result :

$$
P(\varepsilon)=\frac{1}{\varepsilon} e^{-\varepsilon / \bar{\varepsilon}}
$$

The characteristic function $K(\omega)$ of random slivers is calculated by substituting formula (14) into formula (20) as follows:

$$
K(\omega)=\frac{1-j \omega \bar{\varepsilon}}{1+\omega^{2} \bar{\varepsilon}^{2}}=\frac{1}{1+\omega^{2} \varepsilon^{2}}-\frac{j \omega \bar{\varepsilon}}{1+\omega^{2} \bar{\varepsilon}^{2}}
$$

The spectral density function of random slivers is then deduced as follows:

If formula (21) is substituted into formula (17), then

$$
\begin{aligned}
\phi(\omega)-\phi^{2}(\omega) & -\psi^{2}(\omega)=\frac{1}{1+\omega^{2} \bar{\varepsilon}^{2}} \\
& -\frac{1}{\left(1+\omega^{2} \bar{\varepsilon}^{2}\right)^{2}}-\frac{\omega^{2} \varepsilon^{2}}{\left(1+\omega^{2} \bar{\varepsilon}^{2}\right)^{2}}=0
\end{aligned}
$$
becomes

Hence, the spectral density function $Y(\omega)$

$$
Y(\omega)=\frac{4 a^{2}}{\varepsilon \omega^{2}}(1-R e \cdot Z(\omega))
$$

where $Z(\omega)$ has already been defined by formula (15). In other words, we can get the characteristic function $Z(\omega)$ if the fiber length distribution is given.

The mean distance between the leading ends of fibers, $\bar{\varepsilon}$, is given as follows:

$$
\bar{\varepsilon}=\frac{L a}{A}
$$

where $L \quad$ : mean fiber length
$A$ : mean thickness of sliver

$a$ : thickness of a single fiber

For instance, using denier as the unit of $a$, we must use denier as the unit of $A$.

Substituting formula (23) into (22) gives us:

$$
Y(\omega)=\frac{4 a A}{L \omega^{2}}(1-R e \cdot Z(\omega))
$$

$X(p)$ is easily deduced by substituting formula (24) into (6) with $\omega=2 \pi / p$ :

$$
X(p)=\frac{a A}{\pi^{2} L}\left(1-R e \cdot Z\left(\frac{2 \pi}{p}\right)\right)
$$

\section{1-5. Blending}

Let us consider random slivers where different staples are blended (see Figure 6). Here we assume that the spinning process and the blending are carried out perfectly. The conditions of the blending are indicated in Table 1. Then, the spectral density function of the blended slivers is as follows:

$$
Y(\omega)=\frac{4 A}{\omega^{2}} \sum_{r=1}^{n} \frac{a_{r} K_{r}}{L_{r}}\left(1-R e \cdot Z_{r}(\omega)\right)
$$

By formulas (26) and (6), $X(p)$ becomes:

$$
X(p)=\frac{\mathrm{A}}{\pi^{2}} \sum_{r=1}^{n} \frac{a_{r} K_{r}}{L_{r}}\left(1-R e \cdot Z_{r}\left(\frac{2 \pi}{p}\right)\right)
$$

\begin{tabular}{|c|c|c|c|c|c|}
\hline Staple & $\begin{array}{l}\text { Proportions } \\
\text { of blending } \\
\text { (weight) }\end{array}$ & $\begin{array}{l}\text { Thickness } \\
\text { of single } \\
\text { fibers }\end{array}$ & $\begin{array}{l}\text { Probability } \\
\text { distribution } \\
\text { function of } \\
\text { fiber length }\end{array}$ & $\begin{array}{l}\text { Mean } \\
\text { fiber } \\
\text { length }\end{array}$ & $\begin{array}{l}\text { Characteristic } \\
\text { function of } l\end{array}$ \\
\hline $\begin{array}{l}1 \\
2 \\
3\end{array}$ & $\begin{array}{l}K_{1} \\
K_{2} \\
K_{3}\end{array}$ & $\begin{array}{l}a_{1} \\
a_{2} \\
a_{3}\end{array}$ & $\begin{array}{l}P_{1}(1) \\
P_{2}(1) \\
P_{3}(1)\end{array}$ & $\begin{array}{l}\mathrm{L}_{1} \\
\mathrm{~L}_{2} \\
\mathrm{~L}_{3}\end{array}$ & $\begin{array}{l}\mathrm{Z}_{1}(\omega) \\
\mathrm{Z}_{2}(\omega) \\
\mathrm{Z}_{3}(\omega)\end{array}$ \\
\hline
\end{tabular}

\section{Examples of Calculation}

Example 1. Random Slivers of a Uniform Fiber Length

The spectral density of random slivers in the case of fibers of a uniform length is calculated here. In this case, the probability distribution function of fiber length, $P(l)$, is represented by the following formula :

$$
P(l)=\delta(l-L)
$$

where $\delta(l)$ is Dirac's delta function and defined as: 


$$
\delta(l)=\left\{\begin{array}{l}
\infty \text { for } l=0 \\
0 \text { for } l \neq 0
\end{array}\right.
$$

and

$$
\delta(-l)=\delta(l), \int_{-\infty}^{\infty} \delta(l) d l=1
$$

Figures ( 7 a) and ( 7 b) show the staple diagram of this fiber and the distribution function $P(l)$ of fiber length, respectively.

According to formula (15), the characteristic function $Z(\omega)$ is

$$
Z(\omega)=\int_{0}^{\infty} \delta(l-L) e^{-j \omega t} d l
$$

Carrying out the integration indicated, we get:

$$
Z(\omega)=e^{-j \omega L}
$$

Substituting formula (29) into formula (24) gives:

$$
Y(\omega)=\frac{4 a A}{L} \cdot \frac{1-\cos L \omega}{\omega^{2}}
$$
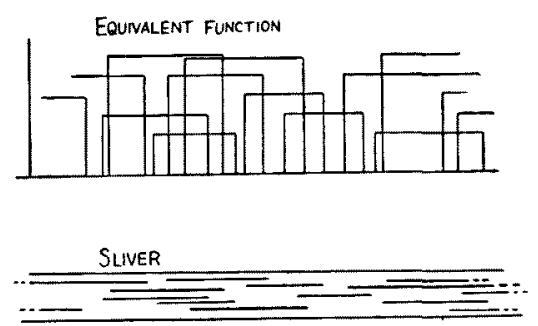

Fig. 6 Blended slivers and their equivalent representation as the assembly of "Single fiber function."

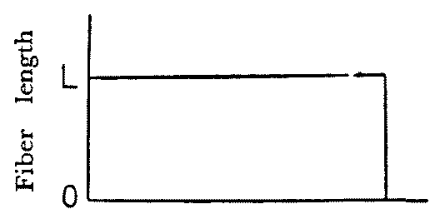

Fig. 7a Staple diagram

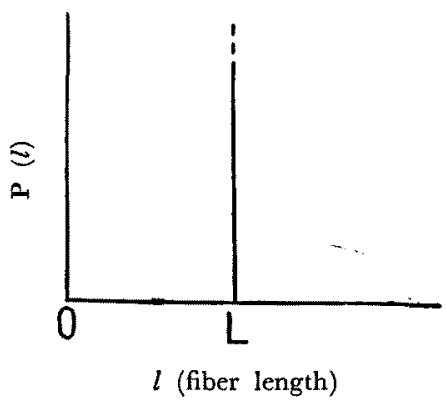

Fig. 7b Probability distribution of fiber length.
This is the spectral density of random slivers where fibers are uniform in length. And $X(p)$ becomes

$$
X(p)=\frac{a A}{\pi^{2} L}\left(1-\cos \frac{2 \pi L}{p}\right)
$$

Let $\mathcal{N}$ be the average number of fibers in the cross section of a sliver, and then the thickness of the sliver is:

$$
A=a N
$$

Therefore, formula (31) is rewritten as follows:

$$
X(p)=\frac{a^{2} N}{\pi^{2} L}\left(1-\cos \frac{2 \pi L}{p}\right)
$$

Figure 8, in which formula (32) is plotted, shows that the variance-the irregularity of slivers-concentrates noticeably in the wave length range which is about twice the fiber length. This phenomenon will often be seen when the Fourier analysis of the irregularity curves of normal yarns is put into practical operation.

Now, let us substitute some practical values into formula (32). Take the spinning conditions shown in Table 2. The $X(p)$ which are the spectrum of variance $\sigma^{2}$ in this case are plotted in Figure 9. We see from it that the characteristics of the irregularity of random slivers are changed considerably by spinning conditions.

Table 2. Spinning Condition.

\begin{tabular}{cccc}
\hline Staple & $\begin{array}{l}\text { Thickness of } \\
\text { single fibers } \\
\text { (den.) }\end{array}$ & Fiber length & $\begin{array}{c}\text { Thickness } \\
\text { of sliver } \\
\text { (den.) }\end{array}$ \\
\hline $\mathrm{a}$ & 3 & $5.08(=2$ in. $)$ & $177\left(=30^{\prime} \mathrm{s}\right)$ \\
$\mathrm{b}$ & 3 & $10.16(=4$ in. $)$ & $177\left(=30^{\prime}\right.$ 's $)$ \\
\hline
\end{tabular}

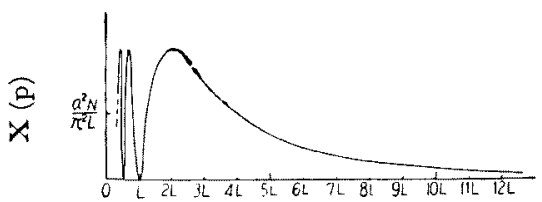

Fig. 8 The spectrum of variance $\sigma^{2}$ corresponding to wave length where fibers are uniform in length.

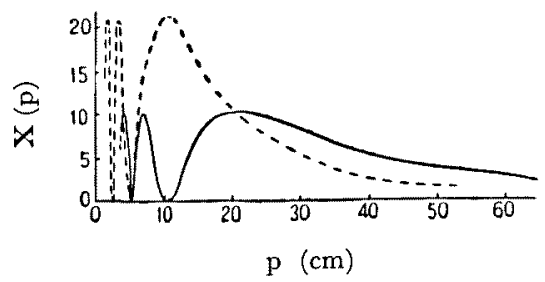

(a) dotted line: fiber length, 2 inches

(b) solid line: fiber length, 4 inches

Fig. 9 Examples of calculation where fibers are uniform in length. 
Example 2. Random Slivers where Fiber Length is Non-uniform and Follows the Normal Distribution

The staple diagram and the probability distribution of fiber length are shown in Figure $10(a, b, c)$ and Figure $11(a, b, c)$, respectively, where case (c) is a special one and means a uniform fiber length.

This type of distribution may be approximately applied to cotton fibers. The spectral density of these random slivers is also calculated by the same method as example 1 .

Assume the fiber length distribution function $P(l)$ to follow the normal distribution function, that is:

$$
P(l)=\frac{1}{\mu \sqrt{2 \pi}} \exp \left\{-\frac{(l-L)^{2}}{2 \mu^{2}}\right\}
$$

where $L:$ mean value of fiber length
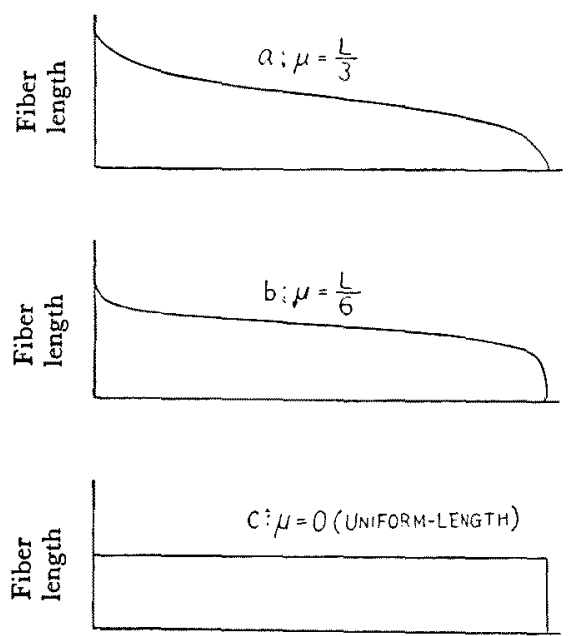

Fig. $10(\mathbf{a}, \mathbf{b}, \mathbf{c})$ Staple diagrams when fiber length follows the normal distribution. $\mu$ is the standard deviation of fiber length. $\mu$ : standard deviation of fiber length and assumedly as follows:

$$
\mu<\frac{L}{3}
$$

From formulas (15) and (33) the characteristic function $Z(\omega)$ is derived as follows:

$$
Z(\omega)=\int_{0}^{\infty} \frac{1}{\mu \sqrt{2 \pi}} \exp \left\{-\frac{(l-L)^{2}}{2 \mu^{2}}\right\} \exp (-j \omega l) d l
$$

For carring out the integration of formula (34), the tequnique of Laplace transformation comes in handy. This integration results in:

$$
Z(\omega)=\exp \left\{-\left(j \omega L+\frac{\omega^{2} \mu^{2}}{2}\right)\right\}
$$

If formula (35) is substituted into (24), the spectral density $Y(\omega)$ is

$$
\begin{aligned}
Y(\omega) & =\frac{4 a A}{\omega^{2}}\left[1-R e \cdot \exp \left\{-\left(j \omega L+\frac{\omega^{2} \mu^{2}}{2}\right)\right\}\right] \\
& =\frac{4 a A}{\omega^{2}}\left(1-e^{-\frac{\omega^{2} \mu^{2}}{2}} \cos L \omega\right) \cdots \cdots \cdots \cdots(36)
\end{aligned}
$$

Hence, the spectrum of variance $\sigma^{2}$ is derived as follows:

$$
X(p)=\frac{a^{2} N}{\pi^{2} L}\left(1-e^{-\frac{2 \pi^{2} \mu^{2}}{p^{2}}} \cos \frac{2 \pi L}{p}\right)
$$

If fiber length is uniform, the value of $\mu$ becomes zero. In this case:

$$
X(p)=\frac{a^{2} N}{\pi^{2} L}\left(1-\cos \frac{2 \pi L}{p}\right)
$$

This formula agrees with the result given in example 1 .

Figures $10 \mathrm{a}, 10 \mathrm{~b}$ and $10 \mathrm{c}$ show staple diagrams corresponding to $\mu=L / 3, L / 6$ and 0 , respectively.

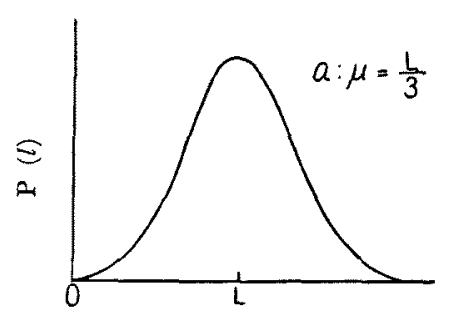

$l$ (fiber length)

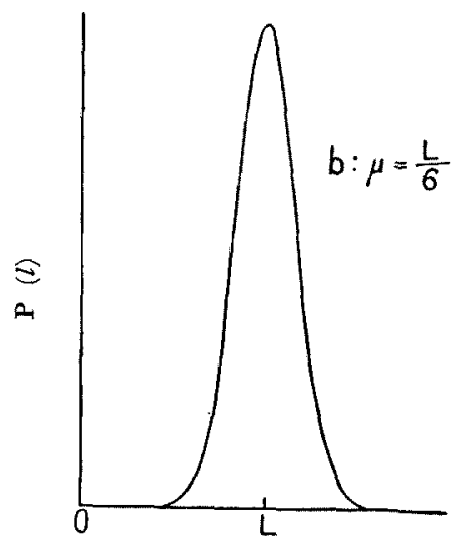

$l$ (fiber length)

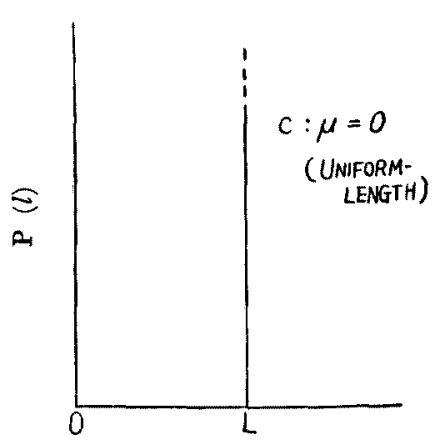

$l$ (fiber length)

Fig. $11(a, b, c)$ Probability distribution functions of fiber length. (Corresponding to Fig. $10(a, b, c)$ ) 
The corresponding probability distribution functions of fiber length are shown in Figures $11 \mathrm{a}, 11 \mathrm{~b}$ and $11 \mathrm{c}$.

Three $X(p)$ curves corresponding to $\mathrm{a}, \mathrm{b}$ and $\mathrm{c}$ above are shown in Figures $12 \mathrm{a}, 12 \mathrm{~b}$ and $12 \mathrm{c}$. Figure $12 \mathrm{c}$ is identical with the result given in example 1 .

As the parameter $\mu$ gets smaller, so irregularity concentration in the specified wave length, $2 L$, becomes more noticeable, especially at $\mu=0$.

Lxamplc 3. Random Slivers where Fiber Lcngth Distribution is a Rectangular Distribution

For this example, we consider a case where the fiber length distribution is a rectangular distribution, i.e., where the staple diagram is a right-angle triangle in form. Figures $13 \mathrm{a}$ and $13 \mathrm{~b}$ are the staple diagram and the probability distribution, respectively, of fiber length.

Fiber length probability distribution function $P(l)$ is formulated as follows:

$$
P(l)=\left\{\begin{array}{l}
\frac{1}{L_{m}} \text { for } 0<l<L_{m} \\
0 \text { for } l<0, \quad l>L_{m}
\end{array} .\right.
$$

where $L_{m}$ : maximum value of fiber length $l$.

Then, the characteristic function $Z(\omega)$ becomes:

$$
Z(\omega)=\frac{1}{j \omega L_{m}}\left(\mathbf{1}-e^{-j \omega L_{* \pi}}\right)
$$

The spectral density function $Y(\omega)$ is
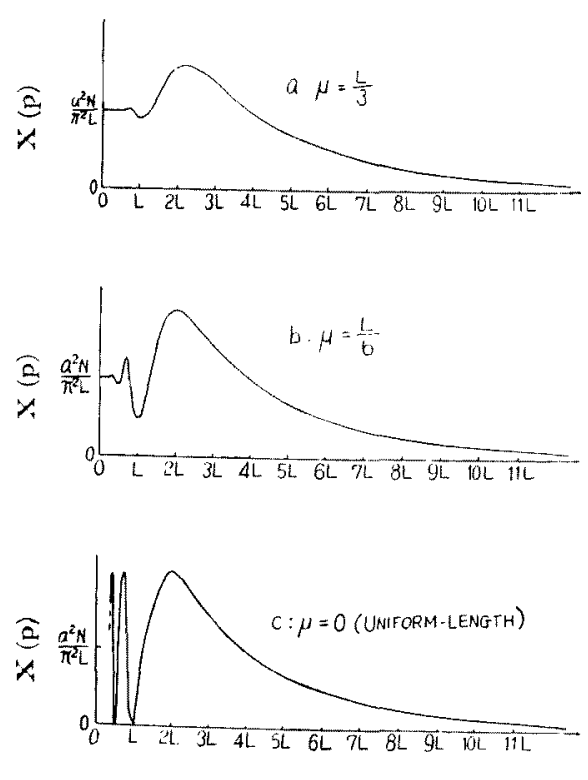

p

Fig. $12(\mathbf{a}, \mathbf{b}, \mathbf{c})$ The spectrum of variance $\sigma^{2}$ when fiber length follows the normal distribution. (Corresponding with Fig. 11 $(a, b, c)$.) L: mean length of fiber.

$$
Y(\omega)=4 a^{2}\left\{\begin{array}{c}
1-\sin L_{m} \omega \\
\bar{\varepsilon} \\
\omega L_{m} \\
\omega^{2}
\end{array}\right.
$$

Let $L$ be the mean fiber length, then $\varepsilon$ is

$$
\bar{\varepsilon}=\frac{L}{N}=\frac{L_{m} / 2}{A / a}=\frac{a L_{m}}{2 A}
$$

From formulas (40) and (41), the $X(p)$ is derived as lollows:

$$
X(p)=\frac{a^{2} N}{\pi^{2} L}\left(1-\frac{p}{4 \pi L} \sin \begin{array}{c}
2 \pi L \\
p
\end{array}\right)
$$

Formula (42) is plotted in Figure 14 which is comparably smooth, with the sharp peaks gone.

\section{Some Features of Random Slivers}

3-1. Changes in the Spectral Density Function Resulting from Ideal Drafting

Consider a drafting system and assume that it gives ideal drafting. By "ideal drafting" we mean drafting which accelerates fibers from input roller speed to output roller speed as soon as their leading ends reach a particular point refered to as the velo-

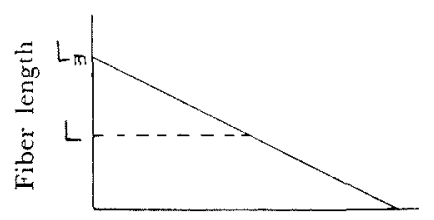

Fig. 13 a The staple diagram when fiber length follows a rectangular distribution.

$$
\mathrm{L}_{\mathrm{m}} \text { : maximum length, } \mathrm{L} \text { : mean length }
$$

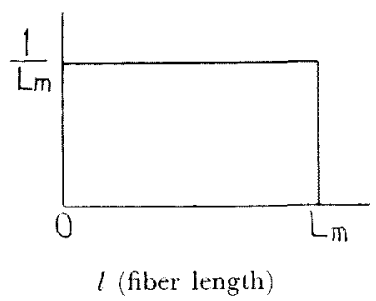

Fig. 13 b The probability distribution when fiber length follows a rectangular distribution.

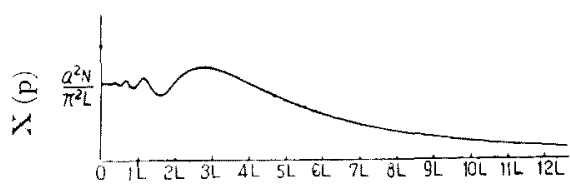

P

Fig. 14 The spectrum of variance $\sigma^{2}$ when fiber length follows a rectangular distribution. L: mean length of the fiber. 
city-change point.

By drafting a random sliver by this system, it is possible to calculate the spectral density of the output sliver from the spectral density function of the input sliver. That is,

$$
Y_{0}(\omega)=\frac{1}{D} Y_{i}(\omega)
$$

where $Y_{i}(\omega)$ : spectral density function of input sliver

$Y_{0}(\omega)$ : spectral density function of output sliver

$D$ : draft.

Thus, there is no difference between pre-drafting spectral density and post-drafting spectral densityexcept in their intensity. The wave length contained in an input sliver is not multiplied by draft. This is one of the most important characteristics of random slivers.

3-2. Changes in Spectral Density Caused by Doubling

Consider some random slivers whose spectral density functions are such as $Y_{1}(\omega), Y_{2}(\omega), \quad r_{3}(\omega)$, $\ldots \ldots Y_{r}(\omega), \ldots \ldots Y_{n}(\omega)$.

Put these random slivers together, and the spectral density function of the combination (or the "combined sliver") becomes as follows:

$$
Y(\omega)=\sum_{r=1}^{n} Y_{r}(\omega)
$$

Thus, a combined sliver is also a random sliver. Formula (44) is applicable to a wide variety of analyses.

Note that these two formulas, (43) and (44), are applicable only to random slivers or random components in actual slivers.

3-3. Total Value of the Irregularity of Random Slivers

We have deduced the functions $X(p)$ of the random slivers of all forms. As defined in formula (7), the function $X(p)$ is the distribution function of variance $\sigma^{\prime \prime}$ corresponding to wave length $p$, and the total area under the curve of $X(p)$ in Figure 4 gives the variance $\sigma^{2}$ of the irregularity of random slivers. That is:

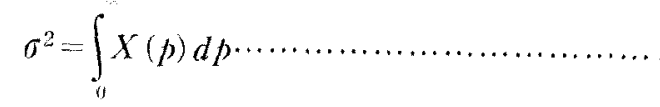

With fibers of a uniform in length, variance $\sigma^{2}$ is calculated by substituting formula (31) into (45):

$$
\sigma^{2}=\lim _{p \rightarrow 1} \int_{11}^{p} \frac{a^{2} N}{\pi^{2} L}\left(1-\cos \begin{array}{c}
2 \pi L \\
p
\end{array}\right) d p \ldots \ldots \ldots
$$

This integration can be calculated by translating the variable into $2 \pi / z$, where $z$ is a complex number.

The result is

$$
\begin{aligned}
& \sigma^{2}=a^{2} N \\
& \quad \sigma=a \sqrt{N}
\end{aligned}
$$

The coefficient of variation, $C V$, is

$$
C V=\frac{\text { standard deviation }}{\text { mean thickness of sliver }}
$$

$$
=\frac{a \sqrt{ } N}{a N}=\frac{1}{\sqrt{N}}
$$

This quantity agrees with the results deducible in the following way: Assuming that the number of fibers in the cross section of a sliver is varied according to Poisson's distribution, the coefficient of variation of this sliver irregularity becomes $1 / \sqrt{N}$. If the fiber length is not uniform, it can be proved that variance $\sigma^{2}$ is also equal to $a^{2} \mathcal{N}$. Hence, the coefficient of variation, $\mathrm{CV}$, is also:

$$
C V=\frac{1}{1 / N}
$$

This is the total quantity of the irregularity which contains the full component of wave length. Furthermore, we can, by the theory discussed in this article, express the irregularity of random slivers as the function of wave length.

For example, consider a random sliver consisting of fibers of a uniform length. Let $C V\left(p_{1}, p_{2}\right)$ be a component of the irregularity $C V$ of this sliver and be defined as the irregularity contained in the wave length range from $p_{1}$ to $p_{2}$. Then, $C V\left(p_{1}, p_{2}\right)$ is the function of $p_{1}$ and $p_{2}$ and is expressed thus:

$$
C V\left(p_{1}, p_{2}\right)=\frac{1}{\sqrt{N}} \sqrt{\frac{1}{\pi^{2} L} \int_{b_{1}}^{\beta_{2}}\left(1-\cos \frac{2 \pi L}{p}\right) d p}
$$

This is the generalized representation of formula (47) concerning the characteristics of irregularity where fibers are uniform in length.

If fiber length is non-uniform and follows a specified distribution, we can express formula (48) in a different form and get the function $\mathrm{CV}$ $\left(p_{1}, p_{2}\right)$ correspondingly by using the theory we have introduced.

\section{Conclusions}

The characteristics of sliver irregularity where fibers are distributed according to some statistic distribution in a sliver, have been explained theoretically by means of the spectral density or the spectrum of variance $\sigma^{2}$ corresponding to wave length. As a special case of this fundamental theory, the character- 
istics of the so-called random slivers have been explained.

The characteristics of random slivers arc changed by, among other things, the distribution of fiber length, the thickness of slivers, the thickness of single fibers, and the conditions of blending. Some examples of changes have been shown by calculations. These examples show many interesting facts, such as, for instance, that when fiber length is uniform, the most remarkable wave length contained in its irregularity is $2 L$ where $L$ is the fiber length. This phenomenon will be seen when the Fourier analysis of the irregularity of a normal yarn is carried out in practice.

On the other hand, in designing spinning conditions for some fibers, or in designing conditions for their blending, we can-assuming that the spinning process will be carried out perfectly-predict the characteristics of the irregularity of the final yarns.

The theory introduced in this article can be applied in solving many theoretical and practical problems, including, for example, the following problem: In comparing practical irregularity and theoretical irregularity to get information about the drafting process, we use, for instance, the following index, known as Huberty's $h:[3]$

$$
K=\frac{C V_{p}}{C V_{T}}
$$

where $C V_{p}$ : practical irregularity
$C V_{T}$ : irregularity of random slivers (or theoretical irregularity) As the value of $C V_{T}, 1 / \mathcal{N}$ is adopted where $\mathcal{N}$ is the avcrage number of fibers in the cross section.

However, $C V_{p}$, being given by indication by an evenness tester, is not the quantity of irregularity containing the full component of wave length, because the frequency response of an evenness tester is limited. It is unreasonable to compare $C V$, with $C V_{T}$, because $C V_{T}$ is the quantity of theoretical irregularity which contains the full wave length component. If, however, the frequency response of an evenness tester is known, the corrected value for $C I^{*}$ can be calculated by the spectral density function of this random sliver.

Furthermore, the theory under review can be applied in solving problems encountered in formulating a drafting theory based on random slivers, the theory of blending and automatic control of sliver levelness.

\section{References}

[1] H. C. Picard: 7. Text. Inst., Vol, 42, T. 503 (1951)

[2] H. S. Tsien: "Engineering Cybernetics," Mc-Craw Hill, L. S. A., (1954)

$[3]$ R. Temmerman, I. Hermanne; 7. Text. Inst., Vol, 41, T. $411(1950)$ 